\title{
Exploration of miRNAs in Neolamarckia cadamba and their potential cross-kingdom functions
}

\author{
Jia-Jian He', Rui Ye ${ }^{1}$, Ting Chen ${ }^{1}$, Qian-Yun Xi ${ }^{1}{ }^{2}$, Jun-Yi Luo ${ }^{2}$, Hao-Jie Zhang ${ }^{2}$, Jia-Han Wu', Jia-Jie Sun ${ }^{2 *}$ and \\ Yong-Liang Zhang ${ }^{1,2^{*}}$
}

\begin{abstract}
Neolamarckia cadamba (Roxb.) Bosser (Rubiaceae) is a widespread plant with medicine value and has been used for treating various diseases, such as coughs, fevers, anemia, blood disorders and stomach pains. It had been reported that plant miRNAs might enter mammalian intestines and exert a special ingredient across the different species. However, the knowledge about miRNAs in N.cadamba is scarce. In this study, we first applied high-throughput sequencing to identify miRNAs in N.cadamba. Sequencing revealed a total of 11,285,965 raw reads in the small RNA library of N.cadamba leaves. By bioinformatics analysis, we identified a total of 192 miRNAs, including 167 conserved miRNAs and 25 novel miRNAs, which were distributed among 30 families. Next, we used miRanda to predict the targets of those miRNAs in human, 4030 target genes were predicted. Furthermore, Gene ontology (GO) annotation and Kyoto Encyclopedia of Genes and Genomes (KEGG) pathway analyses indicated that the identified miRNAs majored in binding, vibrio cholerae infection and insulin signaling, which were likely to provide valuable references for further understanding of medical functions of $N$. cadamba.
\end{abstract}

Keywords: Neolamarckia cadamba, High-throughput sequencing, miRNAs analysis

\section{Introduction}

The $N$. cadamba is a member of the tribe Naucleeae in the family Rubiaceae and distributed widely in South Asia and the south of China [28]. According to present data, $N$. cadamba as a representative medicinal plants which is widely known as India Ayurvedic system of Medicine (IASM) [15] The studies of N. cadamba have mainly been focused on its medicinal values in the treatment of eye infections, antidiarrheal, skin diseases, dyspepsia and the stomatitis, coughs, fevers, anaemia, blood disorders and stomach pains $[4,26]$. The identified major active components in bark and leaf are alkaloids, saponins, flavoniuds, terpenoids and chlorogenic acids [20]. Until now, scientific bioactivity identification researches have uncovered its antimalarial [30], anti-hepatotoxic [20], anti-inflammatory

\footnotetext{
*Correspondence: jiajiesun@scau.edu.cn; zhangyl@scau.edu.cn

${ }^{1}$ Guangdong Province Key Laboratory of Animal Nutritional Regulation, 483 Wushan Road, Tianhe District, Guangzhou 510642, Guangdong, China

${ }^{2}$ National Engineering Research Center for Breeding Swine Industry, College of Animal Science, South China Agricultural University, 483 Wushan Road, Tianhe District, Guangzhou 510642, Guangdong, China
}

[9], antioxidant, wound healing and antimicrobial activities [31]. Narayan's group found that the application of defatted methanol extract $N$. cadamba (MENC) on Ehrlich ascites carcinoma (EAC) played a constructive role on antitumor activity and antioxidant activity in vivo [13].

MicroRNAs (miRNAs) are a class small non-coding RNAs, which are 18-24 nucleotide in length and inhibit gene expression by mRNA cleavage or translation repression in the $3^{\prime}$ untranslated region (3'UTR) [8]. They are crucial on gene expressions, and a number of articles indicated that miRNAs not only execute biological functions inherent in their system, but can also be transferred from one species to another. Recent studies have shown that in addition to regulating intracellular levels, microRNAs also affect intercellular levels, even in interspecies [11, 23]. For instance, several years ago, plant microRNAs were discovered in sera and tissues of human and other animals [35]. Further studies showed these plant microRNAs were absorbed from food; one that was relatively high in serum, miR168a, directly targeted low-density lipoprotein receptor adaptor protein 1 
(LDLRAP1) in liver cells and decreased the clearance of LDL from the blood. In 2015, Zhen et al. found plant miRNA miR2911 (Lonicera japonica, Honeysuckle) can directly target influenza A viruses to inhibited H5N1 and H7N9 viral replication [36]. Derived plant miR159 significant inhibited breast cancer cell proliferation by targeting transcription factor 7 (TCF7) [12]. An ever increasing number of researches indicated that plantengineered miRNAs can be absorbed in the mammalian digestive tract and act as a bioactive constituen to target mammalian genes to mediating cross-kingdom regulations [24, 25, 34].

It is well known that a wide range of biological activities are in the N.cadamba And these substances play an important role, Due to a large number of studies showing that plant miRNAs can regulate biological activity across boundaries, miRNAs of $N$. cadamba may also play a certain role in addition to various roles of active ingredients, however, miRNAs of N.cadamba still remain unknown. Because the yield of huangliang wood leaves is large and easy to get, we choose leaves as raw materials, In the present study, we used high-through put sequencing and qPCR, and identified conserved and novel miRNAs in N.cadamba leaves, and further analyzed the miRNAs functions in N.cadamba via bioinformatics analysis. Next, we used miRanda to predict the targets of those miRNAs in human. This is the first reporte about miRNAs of N.cadamba and will Provide foundation for further understanding of active components in herbs.

\section{Results}

Summary of small RNA library data via deep sequencing Total RNA was extracted and a small RNA library was constructed from $N$. cadamba leaves. As a result, a total of 11,285,965 raw reads were obtained. With filtering the adapters and low quality reads, 10,878,015 clean reads remained (Table. 1). The length distributions of the small RNAs were shown in Fig. 1 and majority of reads were 21 to $24 \mathrm{nts}$ in length, the main size groups were $24 \mathrm{nt}$, The amounts of $24 \mathrm{nt}$ small RNAs were

Table 1 Summary of reads in small RNA libraries in N. cadamba

\begin{tabular}{lll}
\hline Category & Reads & Percent (\%) \\
\hline Total raw reads & 11285965 & $100.00 \%$ \\
Clean reads & 10878015 & $96.39 \%$ \\
Mapped to N. cadamba transcriptome & 3328945 & $29.50 \%$ \\
Known_miRNA & 243319 & $2.16 \%$ \\
Novel_miRNA & 95552 & $0.08 \%$ \\
rRNA & 186054 & $1.65 \%$ \\
SnRNA & 2905 & $0.03 \%$ \\
SnoRNA & 753 & $0.01 \%$ \\
\hline
\end{tabular}

approximately $39.42 \%$. After initial processing, the high quality small RNAs reads were mapped to the N. camdaba leaves transcriptome genome using the bowtie software, and the number of total/unique sequences that paired with the transcriptome were 6,935,424/3,328,945, approximately half $(48 \%)$ of total reads were mapped successfully. Only a small portion of small RNAs could be annotate (530,525 redundant reads, 28,031 unique reads), thus, the unannotated reads were collected for further prediction of novel miRNAs. The top $10 \mathrm{miR}$ NAs occupied a percentage of $68.2 \%$, top 20 miRNAs and top 50 miRNAs has a dominant percentage of 85.9 and $96.4 \%$, respectively (Fig. 2a). Therefore, the miRNAs are highly concentrated in the top 50, as shown in (Fig. 2b), and the top 10 miRNAs were miR159a, miR166u, miR395b, miR395a, miR166a-3p, miR166h-3p, miR166e, novel-1, and novel-4.

\section{Identification and characterization of the conserved miRNAs}

To identify the conserved miRNAs in $N$. cadamba, the small RNA sequences were compared to known plant mature miRNAs in miRBase database (version 21). One hundred sixty-seven known miRNAs that distributed in 30 families were identified. The most abundant conserved miRNAs were miR159 (63,739 reads) and miR166u (55,327 reads), followed by miR395b (48,862 reads) (Additional file 1: Supplemental Table S1). We analyzed miRNA families of $N$. cadamba and found miR-159 and miR-166 represented large families with 19 and 18 members, respectively.

\section{Identified of potentially novel miRNAs in N.cadamba}

As a result, we detected a total of 25 novel miRNAs, with reads varying from 2 to 24,598 reads (Additional file 1: Supplemental Table S1). The lengths of the novel miRNAs ranged from 20 to $24 \mathrm{nts}$, and the precursors ranged from 45 to 279 nts. The most abundant novel miRNAs were novel- 1 with 24,598 reads, followed by novel-4 and novel-8. Although the major novel miRNAs were lower expressed than conserved miRNAs, the species-specific functions they may played must not be overlooked.

\section{Validation of the expression of the predicted miRNAs with RT-PCR}

To further validate miRNAs in N.cadamba, we randomly selected seven miRNAs including five conserved miRNAs and two novel miRNAs for stem-loop qRT-PCR detection. The qRT-PCR results showed that seven miRNAs were detected successfully in samples (Fig. 3) and their abundance were also in consistent with Illumina sequencing results. 


\section{Length Distibution}

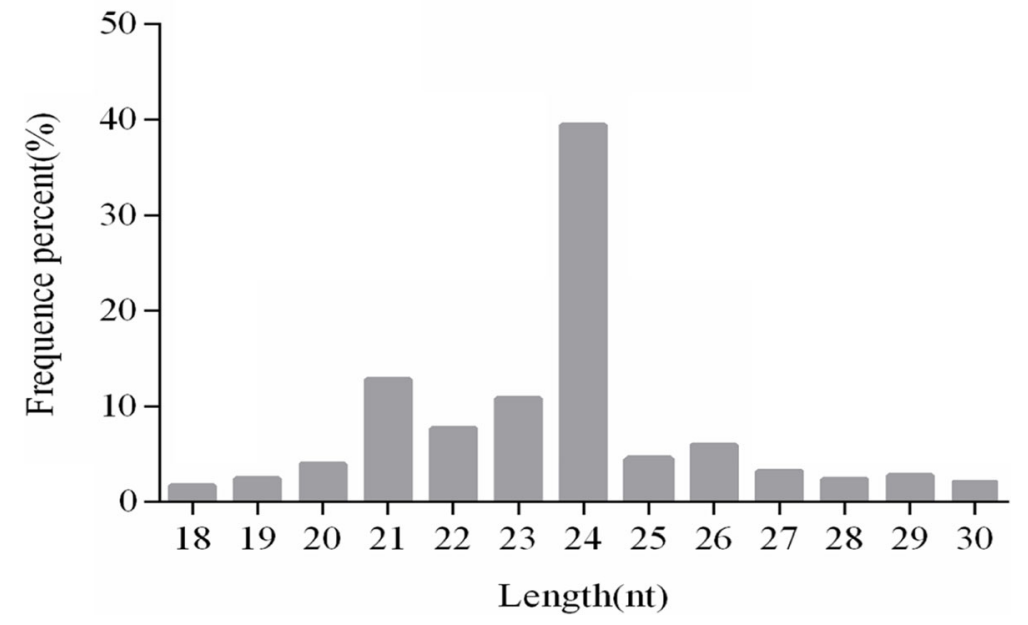

Fig. 1 Length distribution of small RNAs from N. cadamba leaves. A total of 6,935,424 clean reads were obtained, ranging from 18 to $30 \mathrm{nt}$. $24 \mathrm{nt}$ length reads were the most abundant

\section{Prediction of human gene targets for N.cadamba miRNAs}

To investigate whether N.cadamba miRNAs might regulate human gene expression we used miRanda software to search homologous human miRNAs. Result showed 43 miRNAs potentially targeted 4030 genes in human. GO analysis assigned these putative targets into three main categories in terms of biology processes, cellular components, and molecular functions (Additional file 2: Supplemental Table S2). The most enriched GO terms in both species were "cell process" "single-organism cellular process" (BP), "cell", "cell part" (CC), and "binding" (MF). The predicted target genes were mapped to the KEGG database and classified into 279 and 276 pathways of which 18 and 9 signaling pathways were significantly enriched, respectively (Table 2).
As shown in Table 2, 18 of the 43 miRNAs might target about 17 related genes (ATP6VOD2, ATP6VOE1, ATP6V0A4, ATP6V1D, SEC61A2, SEC61A1, PRKCB, PRKCA, PRKACA, ADCY3, ADCY9, ARF1, KDELR3, KCNQ1, PDIA4, AGL, SLC12A2) (Fig. 4a) and are likely to be involved in the vibrio cholera infection in human. In addition, a number of 22(EXOC7, SOCS2, INPPL1, CBL, RPS6KB2, PPARGC1A, IRS1, RPTOR, PPP1R3D, G6PC, TSC1, GSK3B, GYS1, MAPK9, MAPK8, PRKACB, PRKAA2, SHC3, INSR, RAPGEF1, PYGB, FASN) (Fig. 4b) genes targeted by N.cadamba miRNAs were concerned with insulin signaling pathway, and 32 targeted genes may be involved in lipogenesis, glycolysis, anti-lipolysis and glycogenesis process, which contributed to antidiabetic (Additional file 2: Supplemental Table S2).

\section{A}

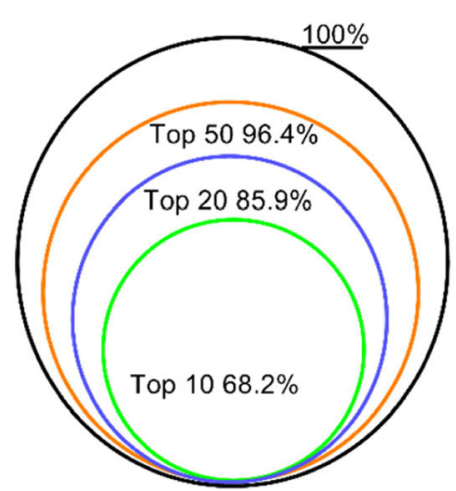

B

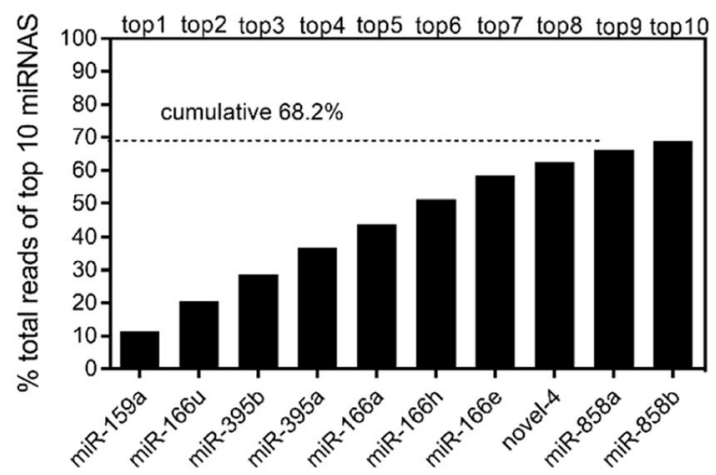

Fig. 2 Distribution of miRNA reads and top 10 miRNAs. a The distribution of miRNA reads showed that top 10, top 20, top 50 and top 100 miRNAs accounted for $68.2,85.9$ and $96.4 \%$ of total reads. b Cumulative proportions of top 10 miRNAs. The miR-159a ranked first, accounting for $10.61 \%$ of total reads. And the miRNA166 family accounted for four of them 


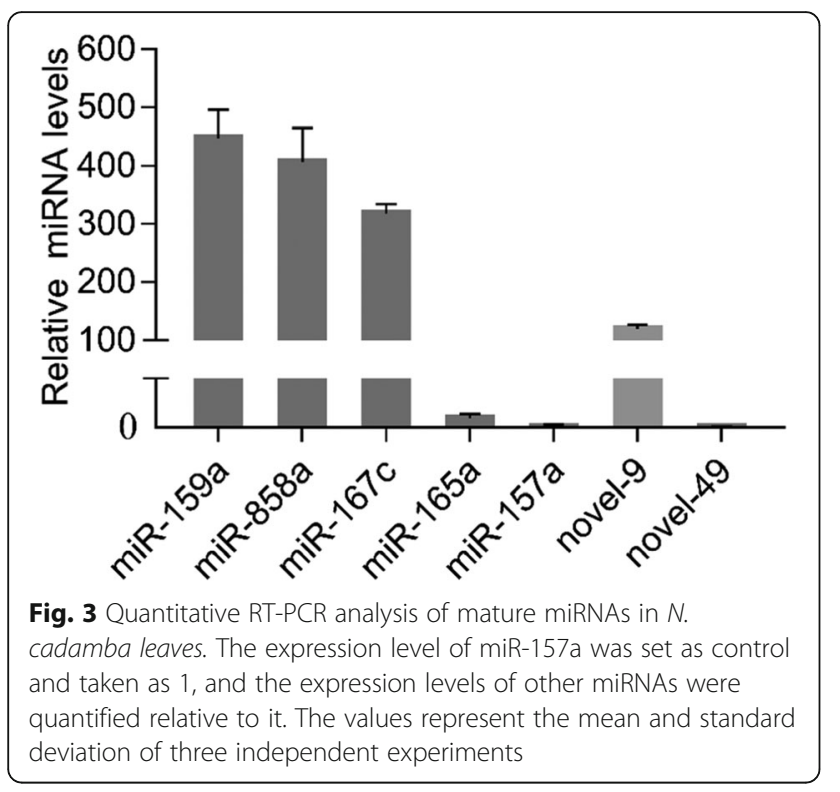

\section{MiR-166e directly targets FASN}

We used miRanda software to identify targets of miR166e in human. A putative binding site conserved among various species was located in FAS (Fig. 5a). To explore this further, a partial FAS normal 3'-UTR sequence and a sequence in which the miR166e binding site is deleted were cloned into a luciferase reporter plasmid (Fig. 5a). HeLa cells were transfected with the reporter plasmids along with synthetic miR166e mimics or NC. The results showed that miR166e significantly reduced luciferase activity, and deletion of the miR166e binding site diminished this reduction (Fig. 5b). These results indicate that miR166e can target FAS.

\section{Discussion}

The miRNAs derived from plants may regulate human genes and have attracted great attention. Zhang and collaborators found rice miRNAs (miR168a) can enter mammalian bloodstream. Later, the miR2911 from honeysuckle were found to target influenza viruses and protect the host. Lately, the miRNA162a can direct target amTOR and influence honeybee caste development [35-37]. The plant miR159 significantly inhibited breast cancer cell proliferation by targeting transcription factor 7 (TFC7) [12]. These articles indicated that plant miRNAs could play a vital role in regulating human health.

N.cadamba was used widely in Indian traditional formulations, and it has been a remedy in the treatments of diarrhea, bacterial infection, and diabetes. However, few studies on the breadth of miRNA has yet been reported for N.cadamba, therefore, the knowledge about molecular mechanisms for compounds produced by N.cadamba is still limited. A total of 167 conserved miRNAs and 25 novel miRNAs were identified. From what has been reported about the medicinal plant Moringa oldifera L, plant miRNAs could regulate cell cycle,

Table 2 Highly enriched KEGG pathways for putative human targets

\begin{tabular}{|c|c|c|c|}
\hline Term & ID & Input number & $P$-Value \\
\hline Vibrio cholerae infection & hsa05110 & 18 & 4.93E-03 \\
\hline Proteoglycans in cancer & hsa05205 & 72 & $1.53 \mathrm{E}-02$ \\
\hline Melanoma & hsa05218 & 30 & 1.86E-02 \\
\hline Pathways in cancer & hsa05200 & 107 & 2.09E-02 \\
\hline Insulin signaling pathway & hsa04910 & 51 & 2.66E-02 \\
\hline Glioma & hsa05214 & 27 & 2.86E-02 \\
\hline ErbB signaling pathway & hsa04012 & 34 & 2.91E-02 \\
\hline Bile secretion & hsa04976 & 29 & 3.17E-02 \\
\hline Aldosterone-regulated sodium reabsorption & hsa04960 & 18 & 3.34E-02 \\
\hline Endocrine and other factor-regulated calcium reabsorption & hsa04961 & 21 & $3.40 \mathrm{E}-02$ \\
\hline Long-term potentiation & hsa04720 & 27 & 3.70E-02 \\
\hline SNARE interactions in vesicular transport & hsa04130 & 16 & 3.86E-02 \\
\hline Focal adhesion & hsa04510 & 69 & 4.14E-02 \\
\hline Bladder cancer & hsa05219 & 17 & 4.61E-02 \\
\hline Synaptic vesicle cycle & hsa04721 & 25 & 4.90E-02 \\
\hline Signaling pathways regulating pluripotency of stem cells & hsa04550 & 49 & $5.12 \mathrm{E}-02$ \\
\hline Thyroid hormone signaling pathway & hsa04919 & 42 & $5.30 \mathrm{E}-02$ \\
\hline Retrograde endocannabinoid signaling & hsa04723 & 37 & $5.58 \mathrm{E}-02$ \\
\hline
\end{tabular}



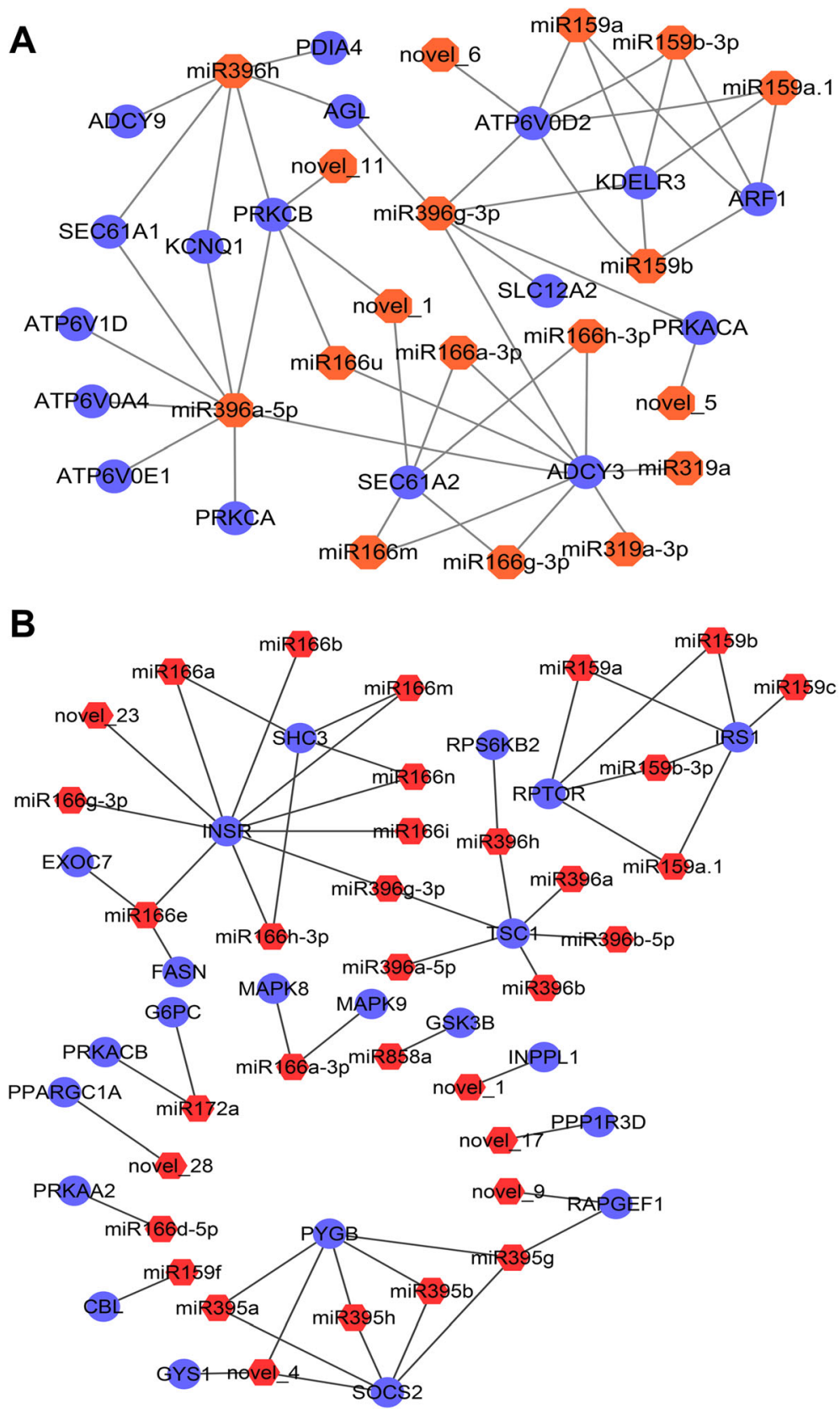

Fig. 4 MiRNAs targeting the genes (In this image, the orange circles show miRNA,the blue circles show target genes). a The 19 miRNAs were analyzed to target 17 vibrio cholera infection genes. b The 34 miRNAs were analyzed to target 22 genes which were concerned with insulin signaling pathway

apoptosis and metabolic in human [27]. Therefore, we predicted the potential targets of N.cadamba miRNAs in human by bioinformatics analysis. The 43 miRNAs of the N.cadamba were targeted to 4030 putative genes in human. GO and KEGG pathways analyses show that those miRNAs may participate in vibrio cholera infection processes.

In human, vibrio cholera infection pathway was highly enrich relative to other pathways. Vibrio cholera is the predominate causes of acute dehydrating diarrhea in developing countries, and the primary virulence factors are cholera toxin (CTX) and toxin-coregulated pilus (TCP), both of which are required for colonization of the human small intestine [14, 19]. Cholera toxin ADP-ribosylates adenylate cyclase, resulting in increased cAMP production and activation of protein kinase A [22, 32].. SLC12A2 can co-express $\beta$-cells, and specific co-expression transporters are known to regulate $\mathrm{Cl}^{-} 、 \mathrm{~K}^{+}$, and $\mathrm{KCNQ} 1$ channels, maintaining the $\mathrm{Cl}^{-}$secretory response by recycling $\mathrm{K}^{+}$entering the cell 


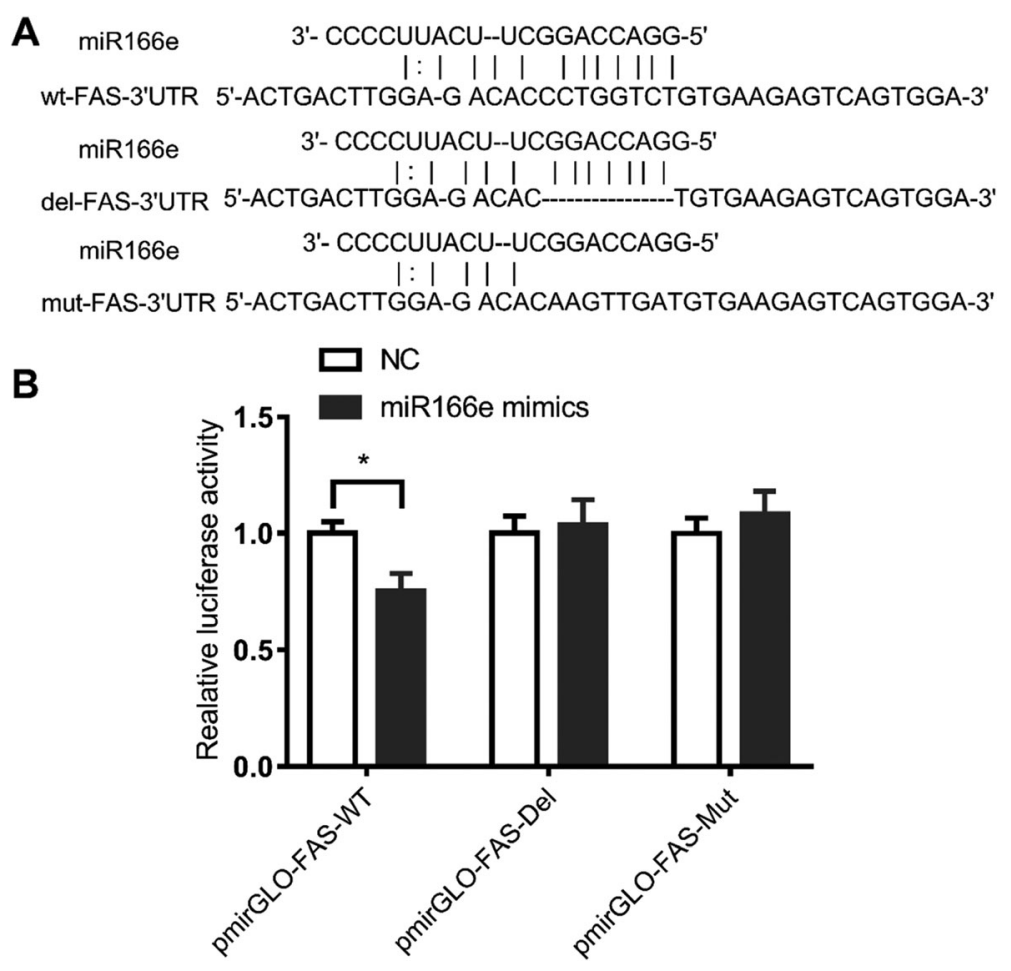

Fig. 5 MiR166e targets FAS. a Prediction of miR166e targets in the FAS 3'-UTR. b HeLa cells were transfected with the pmirGLO-FAS-WT/ pmirGLO-FAS-DEL/ pmirGLO-FAS-MUT luciferase reporters and co-transfected with miR166e mimics or NC mimics. The relative luciferase activity was measured $24 \mathrm{~h}$ later, $n=6$ in each group. Data with error bars mean $\pm \mathrm{SE} *, P<0.05$

$[3,6]$. These relative regulations by vibrio cholerae leads to severe and progressing dehydration and shock. In our study, 18 miRNAs were found involved in vibrio cholera infection. Adenylyl cyclase (AC) was the predicted target of miR166a-3p, miR166u, miR166g, miR166m, miR166h, miR319a, miR396a-5p, miR396g$3 \mathrm{p}$, and miR396h, which can activate cAMP and further influence some key genes that cause diarrhea. The miR396a-5p and miR396h were predicted to target KCNQ1, which were important for $\mathrm{Cl}^{-}$secretion in the colon. An increase in secretion of $\mathrm{Cl}^{-}$, followed by the movement of water, was able to restore the balance of host that results from diarrhea. The SLC12A2, also known as the $\mathrm{Na}^{+}-\mathrm{K}^{+}-\mathrm{Cl}^{-}$co-transporters, was identified as a possible target of miR396g-3p. We hypothesize that some miRNAs identified in N.cadamba leaves regulate vibrio cholera infection by suppressing the processes related to ion transport. However, their regulatory mechanisms needs further studies.

The dynamic regulation of liver glucose metabolism is vital for systemic carbohydrate homeostasis, and the disorder of insulin secretion could lead to diabetes. Previous studies have reported that various extracts of N.cadamba can reduce the blood glucose level of hyperglycemic mice by increasing the glucose uptake $[1,2,5,29]$. Based on our bioinformatics prediction, PI3K/Akt pathway were targeted by N.cadamba miRNAs. The high levels of blood glucose will imbalance the insulin secretion and lead to insulin resistance (IR), which is possibly attributed to a defect in the insulin receptor/IRS-1/PI3K/Akt cascade. IRS-1 was found targeted by miR395b-3p, miR858b, novel-27 and novel-8 in human. IRS-1 tyrosine phosphorylation in response to insulin stimulation, increases the association of IRS-1 with PI3kinase, which leads to activation of serine/threonine kinase protein B (Akt), and p-Akt inhibites the GSK-3 $\beta$ protein directly. The miR159, miR395, miR396 family and novel-1, $4,8,23,28$ miRNAs were predicted to target GSK-3 $\beta$, which phosphorylates and inactivates glycogen synthase (GS) [33]. Therefore, the downstream activated gene encoding GS regulate glucose metabolism through affecting glucose transport and the synthesis of glycogen [17]. Those miRNAs may play a key role in glucose regulation network, and reduce the level of blood glucose. While the direct role of miRNAs in glucose metabolism need to further demonstrated.

\section{Conclusion}

We present the first identification of miRNAs in N.cadamba, and use bioinformatic analysis indicates N.cadamba miRNAs are possibly potential medicinal components involved in regulating mammal targets. Our findings display a foundation for further investigation for 
functions of N.cadamba miRNAs, especially in crosskingdom manner.

\section{Materials and methods Plant material}

The sample of N.cadamba was collected from the College of Forestry, South China Agricultural University. The fresh leaves were immediately frozen in liquid nitrogen and stored at $-80^{\circ} \mathrm{C}$.

\section{RNA extraction}

Frozen tissue was grounded to a fine powder in liquid nitrogen using a mortar and pestle. $100 \mathrm{mg}$ of the powder per sample were transferred into individual RNase-free $1.5 \mathrm{~mL}$ tubes containing $600 \mathrm{~mL}$ of pre-warmed extraction buffer at $60^{\circ} \mathrm{C}$. The extraction buffer consisted of the following: $2 \% \mathrm{CTAB}, 2 \%$ polyvinylpyrrolidone (PVP) $\mathrm{K}^{-40}, 100 \mathrm{mmol} /$ $\mathrm{L}$ TrisHCl (pH 8.0), $25 \mathrm{mmol} / \mathrm{L}$ ethylenediaminetetraacetic acid (EDTA; pH 8.0), $2.0 \mathrm{~mol} / \mathrm{L} \mathrm{NaCl}, 2 \mathrm{~g} / \mathrm{L}$ spermidine and $2 \%$ b-mercaptoethanol (added immediately before use). Lysis buffer without spermidine or b-mercaptoethanol was treated with $0.1 \%$ diethyl pyrocarbonate (DEPC) and autoclaved. $2 \mathrm{~g} / \mathrm{L}$ spermidine was added and the mixture was stored at room temperature. The extracts were mixed by vortexing and incubated at $60^{\circ} \mathrm{C}$ in a water bath for $10 \mathrm{~min}$ with vigorous shaking for several times. Then a third of 5 mol/LKAc ( $\mathrm{pH} 4.8$ ) was added. The supernatant was obtained by ultra-centrifugation at $9000 \mathrm{rpm}$ for $10 \mathrm{~min}$ after an ice water bath for $30 \mathrm{~min}$. An equal volume of chloroform/isoamyl alcohol $(24,1)$ was added to the homogenate and was mixed completely by vortexing. The mixture was centrifuged at $12,000 \mathrm{r} / \mathrm{min}$ for $5 \mathrm{~min}$ at $4{ }^{\circ} \mathrm{C}$. The supernatant was transferred to a new tube with $500 \mu \mathrm{L}$ Phenol Water ( $\mathrm{pH}$ 5.2) and the previous step was repeated. The supernatant was then transferred to a new tube containing an equal volume of isopropyl alcohol for $30 \mathrm{~min}$. RNA is acquired by centrifugal at $4{ }^{\circ} \mathrm{C}$ at $12,000 \mathrm{rpm}$ for $10 \mathrm{~min}$. An equal volume of ethanol was then applied to wash RNA.

\section{Small RNA library construction and deep sequencing}

A total amount of $3 \mu \mathrm{g}$ total RNA per sample was used as input material for the small RNA library. Sequencing libraries were generated using NEBNext ${ }^{\circ}$ Multiplex Small RNA Library Prep Set for Illumina ${ }^{\circ}$ (NEB, USA.) The quantity and purity of total RNA was measured by NanoDrop ND-1000 spectrophotometer (Nano Drop, USA) at $260 / 280 \mathrm{~nm}($ ratio $=2.0)$. The integrity of total RNA was confirmed by Bioanalyzer 2100 and RNA 6000Nano LabChip Kit (Agilent, USA) with RIN number [7.0. Briefly, small RNAs of 18-35 nt in length were first isolated from the total RNA by size fractionation. Then, these small RNAs were ligated with 50-RNA and 30RNA adapters, and subsequently, reverse transcription
PCR was used to create cDNAs. The amplified cDNAs were purified and sequenced with Illumina HiSeq 2500 platform.

After deep sequencing, raw data were processed through Novogene Company's Perl and Python scripts. In this step, clean data were obtained by removing the contaminating reads, sequences containing adapters, without insert tags and reads containing poly $\mathrm{A}$ or $\mathrm{T}$ or $\mathrm{G}$ or C. Sequences from 18 to $30 \mathrm{nt}$ in length were selected for further analysis. Then, the retained reads were searched from NCBI, Rfam, and Repbase database to remove known classes of RNAs (mRNA mRNA, rRNA, tRNA, snRNA, snoRNA, and repeats), so that every unique small RNA is mapped to only one annotation. Since there is no published genome information of N.cadamba, the high-quality reads were mapped to Arabidopsis thaliana genome sequence using the Bowtie software [21]. The mappable small RNA tags were aligned to the miRNA precursor in the miRNA database (miRBase. 21.0; released in June, 2014) to obtain the known miRNA count. Finally, novel miRNAs were predicted by exploring the secondary structure, the dicer cleavage site, and the minimum free energy of the former unannotated small RNA tags which could be mapped to the reference sequence by available software miRDeep 2[16].

\section{Identification of conserved and novel miRNAs}

Mapped small RNA tags were used to look for known miRNAs. MiRBase21. 0 was used as reference, and modified software mirdeep2 and srna-tools-cli were used to obtain the potential miRNAs and draw the secondary structures. Custom scripts were used to obtain the miRNA counts as well as base bias on the first position of identified miRNA with certain length and on each position of all identified miRNA respectively. Those lower than 10 reads were discarded. The characteristics of hairpin structures of miRNA precursors can be used to predict novel miRNAs [7]. The available softwares miREvo and mirdeep2 were integrated to predict novel miRNAs by exploring the secondary structures, the Dicer cleavage sites and the minimum free energy (less than $-18 \mathrm{kcal} / \mathrm{mol}$ ) of the small RNA tags unannotated in the former steps.

\section{Human target gene prediction for the N.cadamba miRNAs} The miRNAs were used for human target prediction. MiRanda was employed to predict putative target genes, and then all the genes were mapped to the KEGG database to identify significantly enriched signal transduction pathways in target genes compared with the whole genome background. 
Validation of miRNAs by stem-loop qRT-PCR

To further verify our identification results, 7 randomly chosen sequences (including 2 novel miRNAs as well as 5 conserved ones) were subjected to stem-loop qRT-PCR. The primers for PCR were listed in (Additional file 2: Supplemental Table S2). The stem-loop qRT-PCR steps as previously described [10]. The novel-49 miRNA was used as the internal control miRNA. The reverse primer for miRNAs was the Uni-miR qPCR Primer offered by the kit One Step PrimeScript miRNA cDNA Synthesis Kit (TaKaRa, Dalian). All reactions were performed in triplicate for each sample. The melting curve was generated to test the specificity of PCR products and avoid the falsepositive peaks. No template control and no reverse transcription control were included in all reactions.

\section{GO and KEGG enrichment analysis}

Gene Ontology (GO) enrichment analysis was used on the target gene candidates of differentially expressed miRNAs ("target gene candidates" in the following). GOseq based Wallenius non-central hyper-geometric distribution, which could adjust for gene length bias, was implemented for GO enrichment analysis. KEGG [18] is a database resource for understanding high-level functions and utilities of biological systems, such as cell, organism and ecosystem, from molecular-level information, especially large-scale molecular datasets generated by genome sequencing and other highthroughput experimental technologies (http://genome. jp/kegg/). We used KOBAS (http://kobas.cbi.pku.edu. $\mathrm{cn} /$ home.do) software to test the statistical enrichment of the target gene candidates in KEGG pathways. The relative correlations among miRNAs and miRNA-target genes in N.cadamba Cytoscape networks were constructed by the Cytoscape 3.6.1.

\section{Dual-luciferase reporter assay}

Based on the predicted miRNA-mRNA binding sequences, normal sequences bearing an miR166e seed binding site or with the FAS 3'-UTR site deleted were generated by two complementary chemically synthesised primers (Sangon Biotech) as follows:

wt-FAS-3'UTR-sense (ACTGACTTGGAGACACCCT GGTCTGTGAGAGTCAGTGGAG)

mut-FAS-3'UTR-sense (ACTGACTTGGAGACACAA GTTGATGTGAAGAGTCAGTGGAG)

del-FAS-3'UTR-sense (ACTGACTTGGAGACACTGT GAAGAGTCAGTGGAG)

The complementary oligonucleotides were resuspended at a 1:1 ratio $(1 \mu \mathrm{g} / \mu \mathrm{L}$ each) in annealing buffer (10 mM TRIS pH 7.5-8.0, $50 \mathrm{mM} \mathrm{NaCl,} 1 \mathrm{mM}$ EDTA) and heated at $95^{\circ} \mathrm{C}$ for $10 \mathrm{~min}$ to denature secondary structure. The temperature was then gradually lowered to room temperature. Annealed products were cloned into the pmirGLO vector (Promega) downstream from the firefly luciferase coding region (between $X h o I$ and XbaI sites). HeLa cells were seeded in 96-well cell culture plates $(3.5 \times 104$ cells per well $)$ and cultured in RPMI 1640 (Life Technologies, Grand Island, NY, USA) with $10 \%$ fetal bovine serum (FBS). The next day, cells were transfected with recombinant pmirGLO-3'UTR vector (100 ng/well) mixed with their corresponding miR166e mimics or NC (3 pmol/well, RiboBio) for $6 \mathrm{~h}$ using Lipofectamine 2000 (Life Technologies). Cells were harvested at $24 \mathrm{~h}$ after transfection, and luciferase activity was detected by a dual luciferase reporter assay system (Promega) according to the manufacturer's recommendations. Normalised firefly luciferase activity (firefly luciferase activity/Renilla luciferase activity) for each construct was compared with that of the pmirGLO vector.

\section{Supplementary information}

Supplementary information accompanies this paper at https://doi.org/10. 1186/s41544-019-0043-8.

Additional file 1: Supplemental Table 1. Statistics of reads by sequencing (Expression profile of conserved and novel miRNAs in N. cadamba).

Additional file 2: Supplemental Table 2. Reverse transcript primers for miRNAs and Highly enriched KEGG pathways for putative human targets.

\begin{abstract}
Abbreviations
AMPK: AMP-activated protein kinase; CTAB: Cetyltrimethyl ammonium bromide; FAS: Fatty acid synthase; GO: Gene ontology; GSK3ß: Glycogen synthase kinase 3 beta; HCE: Houttuynia cordata Thunb extract; KEGG: Kyoto Encyclopedia of Genes and Genomes; LDLRAP1: Low-density lipoprotein receptor adaptor protein 1; miRNAs: microRNAs; N.cadamba: Neolamarckia cadamba; qRT-PCR: quantitative real time RT-PCR; SREBP-1c: Sterol regulatory element binding protein-1C; TCF7: Transcription factor 7
\end{abstract}

\section{Acknowledgements}

None.

Authors' contributions

$J H$ and JS carried out the bioinformatics and analysis, JH, YR and TC participated in drafted the manuscript. QX, $J$ and $M L$ collected the sample and $\mathrm{q}-\mathrm{PCR}$ validation. JW, BZ and JH were performed extract total RNA. YZ conceived of the study and participated in its design and coordination and helped to draft the manuscript. All authors read and approved the manuscript.

\section{Funding}

This work was supported by grants from the National Natural Science Foundation of China [grant numbers 31472163], and The Chinese National Key Scientific Project (2016YFD0500503).

Availability of data and materials

N. cadamba leaf raw sequence reads was deposited (https://www.ncbi.nlm. nih.gov/sra/SRP144809).

Ethics approval and consent to participate Not applicable.

Consent for publication

Not applicable. 


\section{Competing interests}

The authors declare that they have no competing interests.

Received: 12 July 2019 Accepted: 31 October 2019

Published online: 31 January 2020

\section{References}

1. Acharyya S, Dash GK, Mondal S, Dash SK. Studies on glucose lowering efficacy of the Anthocephalus cadamba (Roxb.) Miq. roots. Int J Pharm Bio Sci. 2010;1:1-9.

2. Ahmed F, Rahman S, Ahmed N, Hossain M, Biswas A, Sarkar S, Banna H, Khatun A, Chowdhury MH, Rahmatullah M. Evaluation of Neolamarckia cadamba (Roxb.) Bosser leaf extract on glucose tolerance in glucoseinduced hyperglycemic mice. Afr J Tradit Complement Altern Med. 2011;8: 79-81.

3. Al-Hazza A, Linley J, Aziz Q, Hunter M, Sandle G. Upregulation of basolateral small conductance potassium channels (KCNQ1/KCNE3) in ulcerative colitis. Biochem Biophys Res Commun. 2016;470:473-8.

4. Alam MA, Akter R, Subhan N, Rahman MM, Majumder MM, Nahar L, Sarker SD. Antidiarrhoeal property of the hydroethanolic extract of the flowering tops of Anthocephalus cadamba. Rev Bras Farmacognosia-Braz J Pharmacognosy. 2008;18:155-9.

5. Alam MA, Subhan N, Chowdhury SA, Awal MA, Mostofa M, Rashid MA, Hasan CM, Nahar L, Sarker SD. Anthocephalus cadamba (Roxb.) Miq., Rubiaceae, extract shows hypoglycemic effect and eases oxidative stress in alloxan-induced diabetic rats. Rev Bras. 2011;21:0-0.

6. Alshahrani S, Alvarez-Leefmans FJ, Di Fulvio M. Expression of the Slc12a1 gene in pancreatic beta-cells: molecular characterization and in silico analysis. Cell Physiol Biochem. 2012;30:95-112.

7. Ambros V, Bartel B, Bartel DP, Burge CB, Carrington JC, Chen X, Dreyfuss $G$, Eddy SR, Griffiths-Jones S, Marshall M, Matzke M, Ruvkun G, Tuschl T. A uniform system for microRNA annotation. RNA. 2003;9:277-9.

8. Bartel DP. MicroRNAs: target recognition and regulatory functions. Cell. 2009;136:215-33.

9. Chandrashekar KS, Prasanna KS, Abinash B. Anti-inflammatory effect of the methanol extract from Anthocephalus cadamba stem bark in animal models. Int J Plant Biol. 2010;1:6.

10. Chen T, Xi QY, Ye RS, Cheng X, Qi QE, Wang SB, Shu G, Wang LN, Zhu XT, Jiang $Q Y$, Zhang YL. Exploration of microRNAs in porcine milk exosomes. BMC Genomics. 2014;15:100,

11. Chen X, Liang H, Zhang J, Zen K, Zhang CY. Secreted microRNAs: a new form of intercellular communication. Trends Cell Biol. 2012;22:125-32.

12. Chin AR, Fong MY, Somlo G, Wu J, Swiderski P, Wu X, Wang SE. Crosskingdom inhibition of breast cancer growth by plant miR159. Cell Res. 2016; 26:217-28.

13. Dolai N, Islam A, Haldar PK. Methanolic extract of Anthocephalus cadamba induces apoptosis in Ehrlich ascites carcinoma cells in experimental mice. Indian J Pharmacol. 2016;48:445-9.

14. Faruque SM, Albert MJ, Mekalanos JJ. Epidemiology, genetics, and ecology of toxigenic Vibrio cholerae. Microbiol Mol Biol Rev. 1998;62:1301-14.

15. Fatima N, Ahmad MK, Ansari JA, Khan HJ, Rastogi N, Srivastava SK, Ahmad S, Ali Z. Antiproliferative and antioxidant studies of Anthocephalus cadamba (Roxb.) Miq. Bark. Indian J Pharm Sci. 2016;78:525-31.

16. Friedlander MR, Mackowiak SD, Li N, Chen W, Rajewsky N. miRDeep2 accurately identifies known and hundreds of novel microRNA genes in seven animal clades. Nucleic Acids Res. 2012:40:37-52.

17. Gao S, Guo Q, Qin C, Shang R, Zhang Z. Sea buckthorn fruit oil extract alleviates insulin resistance through the PI3K/Akt signaling pathway in type 2 diabetes mellitus cells and rats. J Agric Food Chem. 2017;65:1328-36.

18. Kanehisa M, Araki M, Goto S, Hattori M, Hirakawa M, Itoh M, Katayama T, Kawashima S, Okuda S, Tokimatsu T, Yamanishi Y. KEGG for linking genomes to life and the environment. Nucleic Acids Res. 2008;36:D480-4.

19. Kaper JB, Morris JG Jr, Levine MM. Cholera. Clin Microbiol Rev. 1995;8:48-86.

20. Kapil A, Koul IB, Suri OP. Antihepatotoxic effects of chlorogenic acid from Anthocephalus cadamba. Phytother Res. 1995;9:189-93.

21. Langmead B, Trapnell C, Pop M, Salzberg SL. Ultrafast and memory-efficient alignment of short DNA sequences to the human genome. Genome Biol. 2009;10:R25.

22. Lencer WI, Tsai B. The intracellular voyage of cholera toxin: going retro. Trends Biochem Sci. 2003;28:639-45.
23. Liang $\mathrm{H}$, Zen $\mathrm{K}$, Zhang J, Zhang $\mathrm{CY}$, Chen $\mathrm{X}$. New roles for microRNAs in cross-species communication. RNA Biol. 2013;10:367-70.

24. Liang H, Zhang S, Fu Z, Wang Y, Wang N, Liu Y, Zhao C, Wu J, Hu Y, Zhang J, Chen X, Zen K, Zhang CY. Effective detection and quantification of dietetically absorbed plant microRNAs in human plasma. J Nutr Biochem. 2015;26:505-12

25. Mlotshwa S, Pruss GJ, MacArthur JL, Endres MW, Davis C, Hofseth LJ, Peña MM, Vance $V$. A novel chemopreventive strategy based on therapeutic microRNAs produced in plants. Cell Res. 2015;25:521-4.

26. Pandey A, Negi PS. Traditional uses, phytochemistry and pharmacological properties of Neolamarckia cadamba: a review. J Ethnopharmacol. 2016;181: $118-35$.

27. Pirro S, Zanella L, Kenzo M, Montesano C, Minutolo A, Potesta M, Sobze MS, Canini A, Cirilli M, Muleo R, Colizzi V, Galgani A. MicroRNA from Moringa oleifera: identification by high throughput sequencing and their potential contribution to plant medicinal value. PLoS One. 2016;11:e0149495.

28. Handa SS, Borris RP, Cordell GA, Phillipson JD. NMR spectral analysis of cadambine from Anthocephalus chinensis. Nat Prod. 1983;46:325.

29. Sanadhya I, Lobo V, Bhot M, Varghese J, Chandra N. Antidiaebetic activity of Anthocephalus Indicus A. Rich. fruits in alloxan induced diabetic rats. Int J Pharm Pharm Sci. 2013;5:519-23.

30. Schwikkard S, van Heerden FR. Antimalarial activity of plant metabolitesElectronic supplementary information (ESI) available: IC50 values of plant metabolites. Nat Prod Rep. 2002;19:675-92 See http://www.rsc.org/ suppdata/np/b0/b008980j.

31. Umachigi SP, Kumar GS, Jayaveera KN, Kishore DVK, Ashok KCK, Dhanapal R. Antimicrobial, wound healing and antioxidant activities of Anthocephalus cadamba. Tradit Complement Altern Med. 2007;4:481-7.

32. Viswanathan $\mathrm{VK}$, Hodges $\mathrm{K}$, Hecht $\mathrm{G}$. Enteric infection meets intestinal function: how bacterial pathogens cause diarrhoea. Nat Rev Microbiol. 2009;7:110-9.

33. Wang LY, Wang Y, Xu DS, Ruan KF, Feng Y, Wang S. MDG-1, a polysaccharide from Ophiopogon japonicus exerts hypoglycemic effects through the PI3K/Akt pathway in a diabetic KKAy mouse model. J Ethnopharmacol. 2012;143:347-54.

34. Yang J, Farmer LM, Agyekum AA, Elbaz-Younes I, Hirschi KD. Detection of an abundant plant-based small RNA in healthy consumers. PLoS One. 2015;10: e0137516.

35. Zhang L, Hou D, Chen X, Li D, Zhu L, Zhang Y, Li J, Bian Z, Liang X, Cai X, Yin Y, Wang C, Zhang T, Zhu D, Zhang D, Xu J, Chen Q, Ba Y, Liu J, Wang Q, Chen J, Wang J, Wang M, Zhang Q, Zhang J, Zen K, Zhang CY. Exogenous plant MIR168a specifically targets mammalian LDLRAP1: evidence of crosskingdom regulation by microRNA. Cell Res. 2012;22:107-26.

36. Zhou Z, Li X, Liu J, Dong L, Chen Q, Liu J, Kong H, Zhang Q, Qi X, Hou D, Zhang L, Zhang G, Liu Y, Zhang Y, Li J, Wang J, Chen X, Wang H, Zhang J, Chen H, Zen K, Zhang CY. Honeysuckle-encoded atypical microRNA2911 directly targets influenza a viruses. Cell Res. 2015;25:39-49.

37. Zhu K, Liu M, Fu Z, Zhou Z, Kong Y, Liang H, Lin Z, Luo J, Zheng H, Wan P, Zhang J, Zen K, Chen J, Hu F, Zhang CY, Ren J, Chen X. Plant microRNAs in larval food regulate honeybee caste development. PLoS Genet. 2017;13: e1006946.

\section{Publisher's Note}

Springer Nature remains neutral with regard to jurisdictional claims in published maps and institutional affiliations.

Ready to submit your research? Choose BMC and benefit from:

- fast, convenient online submission

- thorough peer review by experienced researchers in your field

- rapid publication on acceptance

- support for research data, including large and complex data types

- gold Open Access which fosters wider collaboration and increased citations

- maximum visibility for your research: over $100 \mathrm{M}$ website views per year

At BMC, research is always in progress.

Learn more biomedcentral.com/submissions 